\title{
Kansei Image Evaluation of Teacup based on GA-ELM
}

\author{
Qi Jiang, Li Peng \\ Guizhou University, School of Mechanical Engineering,Guiyang, China \\ 602592930@qq.com
}

Keywords: Cultural products, Teacup, GA-ELM, Kansei image, Design evaluation

\begin{abstract}
In order to improve the design efficiency of cultural product , the Genetic Algorithm-Extreme Learning Machine (GA-ELM) network model is used to evaluate the Kansei image of cultural product. Firstly, the design variables of teacup are analyzed, and then 27 kinds of 3D experimental samples are constructed based on the orthogonal experimental results. Semantic Difference Method (SD method) is used to quantify the samples Kansei image score. The Kansei image evaluation model of GA-ELM cultural products is trained with the design variables of teacup as input parameter and the Kansei image score as output parameter. The reliability of the model is demonstrated by case design. The results show that using GA-ELM model to predict the Kansei evaluation of cultural products has higher feasibility and reliability, can effectively improve the emotional design efficiency of cultural products, and provide direction guidance for design practice.
\end{abstract}

\section{Introduction}

The homogeneous competition of products in the consumer market has intensified, and the consumers demand has accelerated into multiple demands mainly based on emotion and function. Product design with excellent culture as a source not only provides consumers with pleasure and satisfaction, but also guides product diversification and development, enhances market competitiveness, and makes local culture play a unique charm in modern society. However, at this stage, the process of designing cultural products relies on people's own abilities and experiences, and these procedures are relatively flat [1]. There is room for improvement in the application level and efficiency of cultural creativity. At present, the rapid development of computer-aided design technology provides a new choice for improving the efficiency and quality of cultural product design: combining the characteristics of cultural products, and using computer science to quickly and accurately evaluate the Kansei image of cultural products is one of the key methods to solve the problem. Artificial neural network is a kind of machine learning algorithm, which has the advantages of self-learning and non-linear solution. In recent years, scholars at home and abroad have done a lot of research on the application of neural network in product evaluation. Jiang Shan [2] put forward a prediction method based on BP network for matching the buyer's characteristics and automobile's characteristics to solve the problem of non-linear feature matching. Zhu Yan [3] summarized the design elements under the design constraints, and established a BP neural network 
model to fit the relationship between the design elements of the robot head shape and the evaluation of the Kansei image. Guo F [4] established a mobile phone optimization design model based on GA-BP neural network to accurately predict user preference scores. Mei Yi [5] established the GAELM prediction model between die casting process parameters and die grain size, and found the superiority of GA-ELM model. In the above research, the neural network has been deeply studied and improved in the field of product evaluation, but for cultural products, the research is still blank. In view of this, this study takes teacups as the object, considers the particularity of cultural products, and establishes the evaluation model of Genetic Algorithm combined with Extreme Learning Machine (GA-ELM), in order to provide efficient and reliable support for product design through accurate and objective evaluating of product image.

\section{Research process and methods}

\subsection{Research process}

The Study is divided into four stages, as shown in Figure 1. Stage 1: Collecting samples of cultural products and related emotional terms from the market for screening and processing. Stage 2: The design variables of the cultural product are summarized and the orthogonal experiment is used to combine the design scheme. Then establish a three-dimensional sample, and use the semantic difference method to obtain the user's emotional image score of the sample. Stage 3: The GA-ELM network model is established by using MATLAB. The design variables are used as input layer parameters and Kansei scores as output layer parameters to train the neural network. Stage 4: The test 3D samples were built according to the design variables, and the user's Kansei image score and network prediction score were compared to verify the reliability of the model.

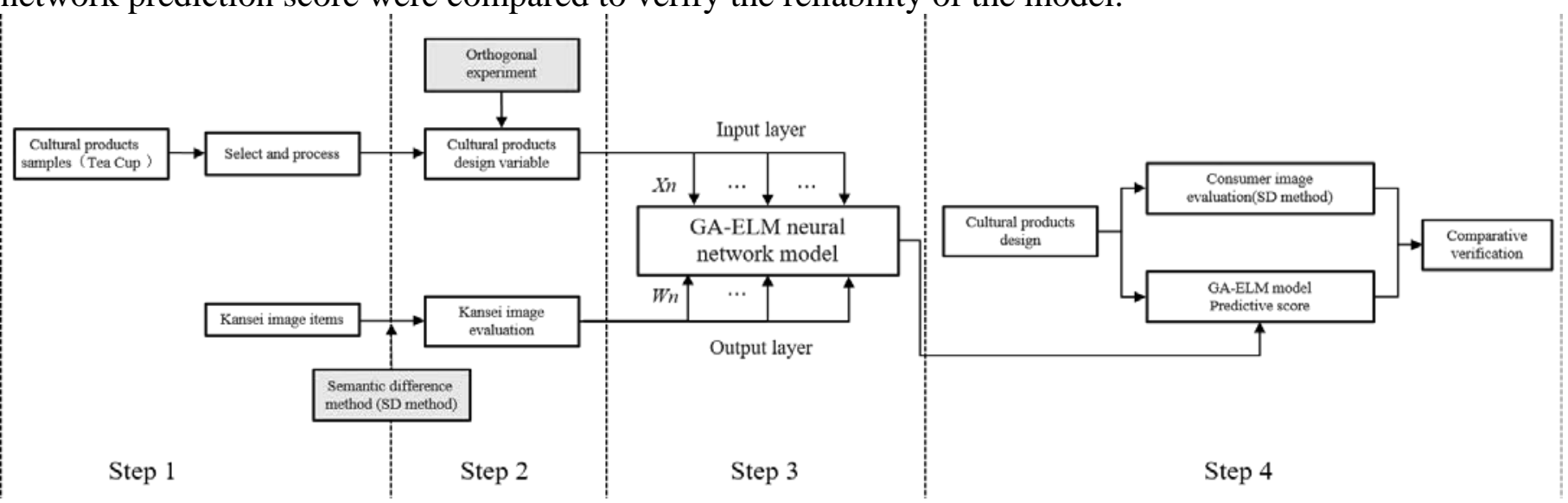

Figure 1. Research process

\subsection{GA-ELM neural network}

Aiming at the shortcomings [6] of general neural network convergence slow, large sample size demand, and easy to fall into local optimum, Huang [7] proposed a new algorithm for single hidden layer feedforward neural network - Extreme Learning Machine (ELM). The only optimal solution can be obtained by setting the number of hidden layer neurons. However, the algorithm randomly generates the connection weights between the input layer and the hidden layer and the threshold of the hidden layer neurons, which makes the hidden layer nodes have the possibility of failure [8]. As a random parallel search algorithm, Genetic Algorithm (GA) has good robustness and global 
indexing ability. And it has excellent global control ability for multi-peak problem. Therefore, GA is used to encode the weights and thresholds in the ELM network. After selection, crossover, and mutation, when the algorithm meets the end condition, the optimal weight matrix and threshold can be obtained [9-10].

Therefore, this study uses MATLAB to build a network model, and uses Genetic Algorithm to optimize the selection of weights and thresholds of Extreme Learning Machine to ensure the efficiency and accuracy of the model. The flow chart is shown in Figure 2.

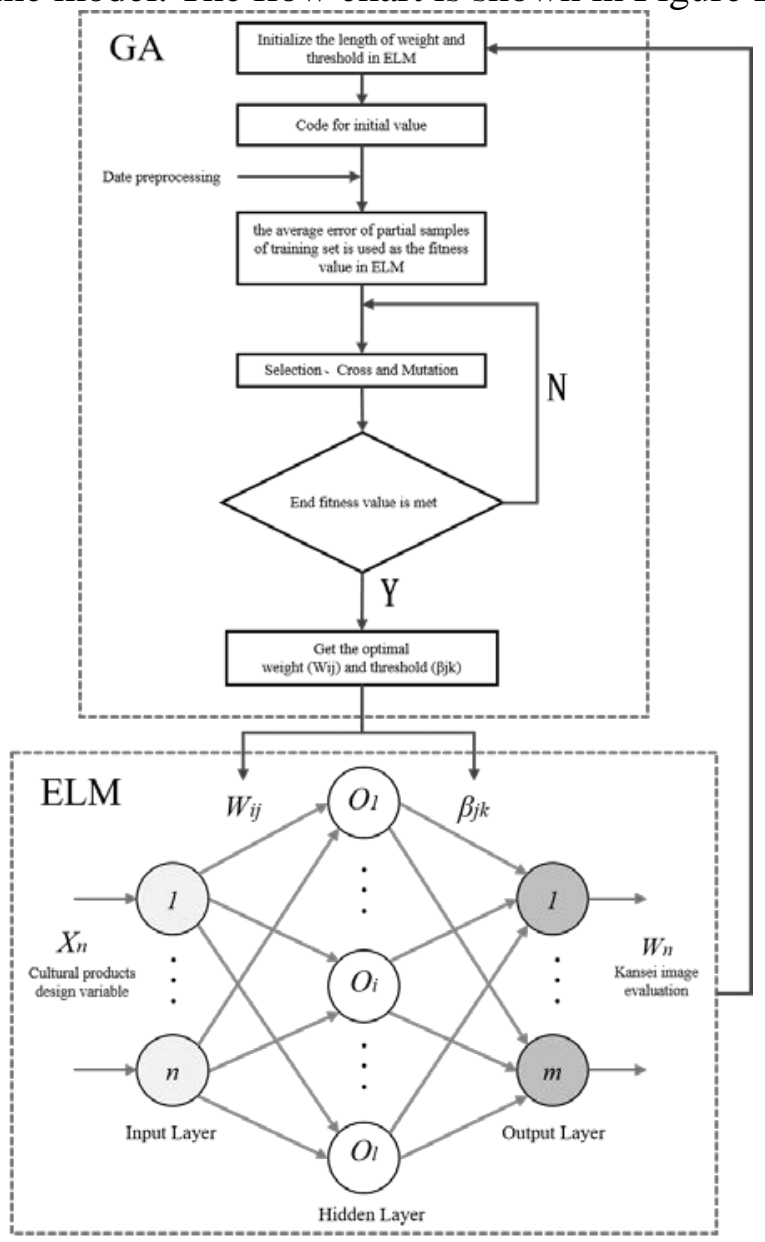

Figure 2. Genetic Algorithm-Extreme learning Machine

\section{Data Acquisition of Neural Network Training}

In this study, the teacup is selected as a case. The design variables of the teacup are used as input parameters and the user's Kansei image scores is used as output parameters to train and validate the model. The research method can also be applied to other cultural products.

\subsection{Design Elements of Teacups}

According to morphological theory, products can be decomposed into several basic components [11]. By combining the basic components, several candidate schemes for different produce modeling can be obtained, including color, texture, lines, detail treatment, composition and meaning, etc. [12]. In this study, the basic structure of the teacup is precisely defined by the size data, and the 
application of the teacup culture is defined in the form of cultural integration. This can accurately describe the Kansei image of the teacup, which is convenient for users to evaluate. Thirty representative teacups collected from the market, including Jingdezhen, Dehua and Liling, were observed and analyzed. From the analysis of the cup forming process, the formation of the cup body can be defined as the side contour is rotated $360^{\circ}$ around the axis. The curvature, direction and shape of the contour curve can define the "high and thin" of the cup. And There are three main forms of cultural application of teacups, including print, carve and decal.

Therefore, the contour curve of teacup is divided into four locating points. The vertical distance between these points and the rotating axis is taken as four variables $\mathrm{X} 1, \mathrm{X} 2, \mathrm{X} 3$ and X4 that affect the shape of teacup, as shown in Figure 3. Taking the cultural application form of teacup as X5, adjusting the style of X5 can affect the cultural temperament of teacup. Finally, the level of teacup elements is summarized as Table1.

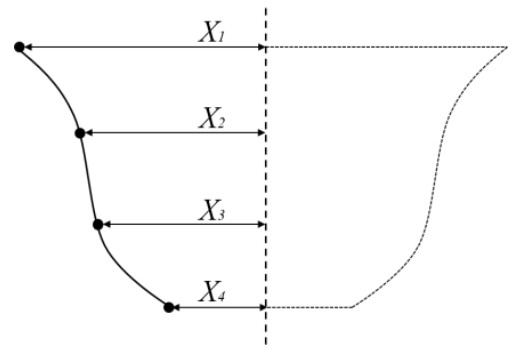

Figure 3. Teacup size variable

Table 1. Factor level

\begin{tabular}{|c|c|c|c|c|c|}
\hline \multirow{2}{*}{ Lever } & \multicolumn{5}{|c|}{ Variable Xn } \\
\cline { 2 - 6 } & $\begin{array}{c}\text { Distance } \\
(\mathrm{X} 1 / \mathrm{mm})\end{array}$ & $\begin{array}{c}\text { Distance } \\
(\mathrm{X} 2 / \mathrm{mm})\end{array}$ & $\begin{array}{c}\text { Distance } \\
(\mathrm{X} 3 / \mathrm{mm})\end{array}$ & $\begin{array}{c}\text { Distance } \\
(\mathrm{X} 4 / \mathrm{mm})\end{array}$ & $\begin{array}{c}\text { Pattern } \\
\text { Type(X5) }\end{array}$ \\
\hline 1 & 180 & 180 & 180 & 140 & 1 Print \\
\hline 2 & 260 & 230 & 215 & 160 & 2 Carve \\
\hline 3 & 320 & 280 & 250 & 180 & 3 Decal \\
\hline
\end{tabular}

The design variables of X1-X5 were selected, and the five elements and three levels orthogonal experiment method, namely L27 $\left(3^{5}\right)$ orthogonal table, was used to design the samples of teacup Kansei evaluation experimental. According to the experimental design results, the Rhino 5.0 software was used to construct three-dimensional experimental samples, and a total of 27 groups of teacup experimental samples were obtained as shown in Figure 4.

\subsection{Kansei image evaluation}

The Kansei image of a product refers to the intuition, Association and feeling of the product form to the user's sensory stimulation, which reflects the user's emotional needs for the product, and is generally described with adjectives [13]. In order to establish an evaluation model for the Kansei evaluation of teacups, it is necessary to obtain the user's Kansei evaluation values for the 27 teacup samples mentioned above. 


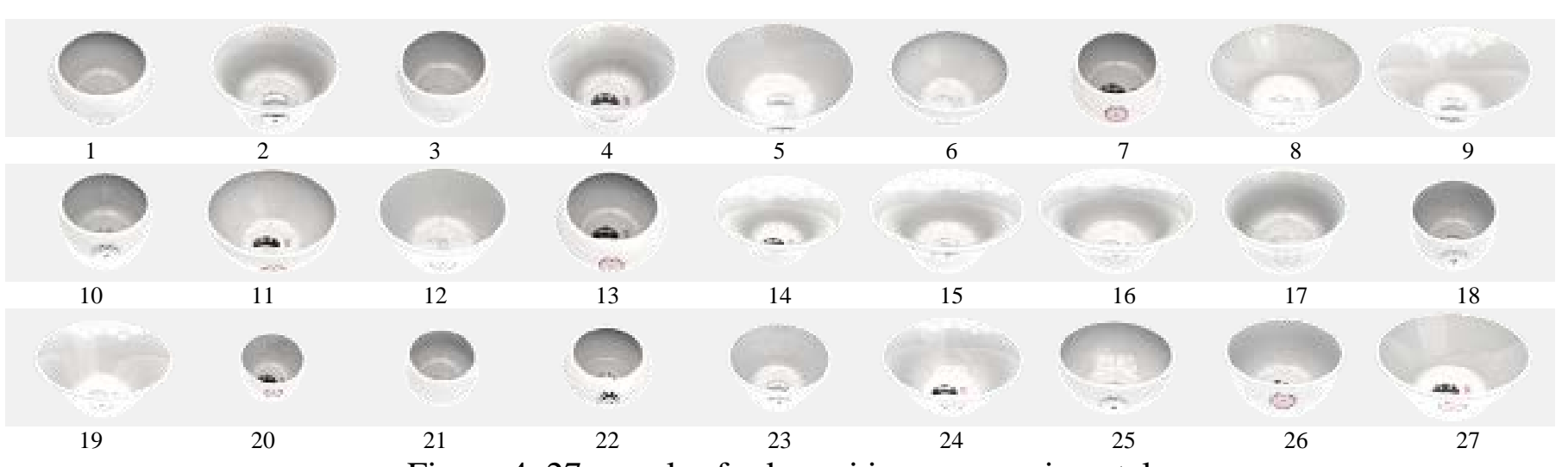

Figure 4. 27 samples for kansei image experimental

Therefore, 76 Kansei items are collected from internet, magazine and dictionary; Using KJ method to sort out, classify, compare and think about the similarity of adjectives; Finally, three Kansei terms describing teacups were determined: beautiful $W_{1}$, thinness $W_{2}$ and luxurious $W_{3}$.

According to three Kansei items, 40 tourism enthusiasts observed 27 experimental samples and scored them. The Kansei image score data were collected using the Likert 5-point scale. The score and the design variable of each sample are summarized as training set in Table 2, which is used as the training sample set of GA-ELM evaluation model.

Table 2. GA-ELM Training set

\begin{tabular}{|c|c|c|c|c|}
\hline \multirow{2}{*}{$\begin{array}{c}\text { Sam } \\
\text { ple }\end{array}$} & \multirow{2}{*}{$\begin{array}{l}\text { Design variable combi- } \\
\text { nation (Input layer) }\end{array}$} & \multicolumn{3}{|c|}{ Kansei image scores (Output Layer) } \\
\hline & & Beautiful $W_{l}$ & Thinness $W_{2}$ & Luxurious $W_{3}$ \\
\hline 1 & $X_{11} X_{23} X_{31} X_{42} X_{52}$ & 1.0 & 2.3 & 1.5 \\
\hline 2 & $X_{12} X_{21} X_{32} X_{43} X_{53}$ & 3.6 & 2.0 & 3.4 \\
\hline 3 & $X_{11} X_{22} X_{32} X_{43} X_{52}$ & 2.8 & 1.3 & 2.1 \\
\hline 4 & $X_{12} X_{21} X_{31} X_{43} X_{51}$ & 3.2 & 2.8 & 3.0 \\
\hline 5 & $X_{13} X_{23} X_{33} X_{43} X_{53}$ & 1.8 & 0.7 & 1.3 \\
\hline 6 & $X_{12} X_{23} X_{31} X_{41} X_{52}$ & 2.9 & 2.4 & 2.9 \\
\hline 7 & $X_{11} X_{22} X_{33} X_{43} X_{51}$ & 4.4 & 2.1 & 3.2 \\
\hline 8 & $X_{13} X_{23} X_{31} X_{43} X_{52}$ & 1.2 & 0.8 & 0.7 \\
\hline 9 & $X_{13} X_{22} X_{31} X_{41} X_{53}$ & 3.3 & 2.7 & 3.7 \\
\hline 10 & $X_{11} X_{22} X_{31} X_{43} X_{53}$ & 3.5 & 2.9 & 4.0 \\
\hline 11 & $X_{12} X_{23} X_{32} X_{41} X_{51}$ & 3.8 & 2.2 & 2.9 \\
\hline 12 & $X_{12} X_{22} X_{32} X_{42} X_{52}$ & 1.8 & 1.7 & 1.7 \\
\hline 13 & $X_{11} X_{23} X_{32} X_{42} X_{51}$ & 3.0 & 2.6 & 2.7 \\
\hline 14 & $X_{13} X_{21} X_{31} X_{42} X_{51}$ & 0.1 & 2.8 & 0.9 \\
\hline 15 & $X_{13} X_{21} X_{32} X_{42} X_{53}$ & 2.3 & 2.1 & 2.7 \\
\hline 16 & $X_{13} X_{21} X_{33} X_{42} X_{52}$ & 1.1 & 1.4 & 1.1 \\
\hline 17 & $X_{12} X_{21} X_{33} X_{43} X_{52}$ & 2.2 & 1.5 & 1.9 \\
\hline 18 & $X_{11} X_{21} X_{32} X_{41} X_{53}$ & 3.3 & 3.7 & 4.4 \\
\hline 19 & $X_{13} X_{22} X_{32} X_{41} X_{52}$ & 2.3 & 1.8 & 2.1 \\
\hline 20 & $X_{11} X_{21} X_{31} X_{41} X_{51}$ & 3.2 & 4.3 & 4.1 \\
\hline 21 & $X_{11} X_{21} X_{33} X_{41} X_{52}$ & 1.7 & 3.0 & 2.5 \\
\hline 22 & $X_{11} X_{23} X_{33} X_{42} X_{53}$ & 2.0 & 1.6 & 2.1 \\
\hline 23 & $X_{12} X_{22} X_{31} X_{42} X_{53}$ & 2.5 & 2.4 & 3.1 \\
\hline 24 & $X_{13} X_{22} X_{33} X_{41} X_{51}$ & 3.7 & 1.8 & 2.6 \\
\hline 25 & $X_{12} X_{23} X_{33} X_{41} X_{53}$ & 2.8 & 1.6 & 2.6 \\
\hline 26 & $X_{12} X_{22} X_{33} X_{42} X_{51}$ & 3.2 & 1.7 & 2.2 \\
\hline 27 & $X_{13} X_{23} X_{32} X_{43} X_{51}$ & 2.8 & 0.6 & 1.0 \\
\hline
\end{tabular}




\section{Training and Verification of GA-ELM Model}

\subsection{Network training}

The GA-ELM network constructed by MATLAB is trained by using the design elements $X_{n}$ of Table 2 as input and the corresponding Kansei image score $W_{n}$ as output, as shown in Fig.5. After adjusting the relevant parameters, 12 hidden layer nodes, 200 training times, the training target error is 0.001 , the number of genetic algorithm population is 96 , and the number of iterations is 300 .

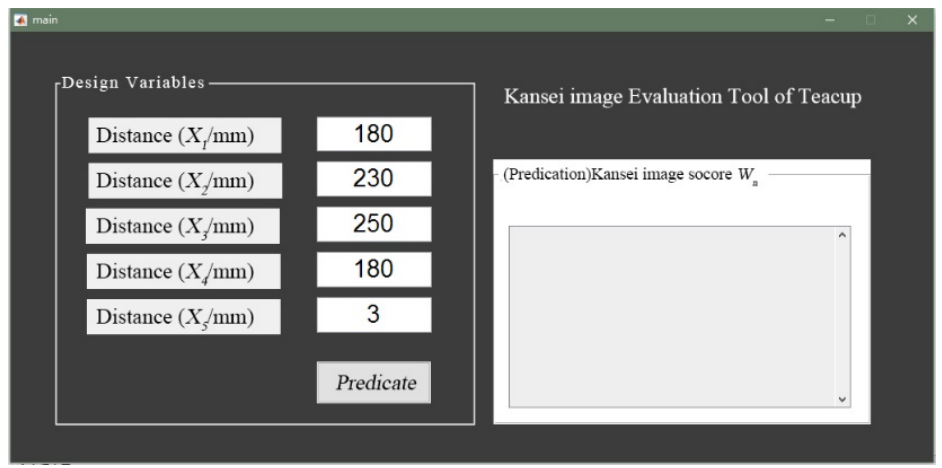

Figure 5. The Kansei image Evaluation Tool interface

\subsection{Network Verification}

In order to verify the validity of the model, three groups of test samples were made according to the combination parameters of the elements in Table 1 (Fig. 6).

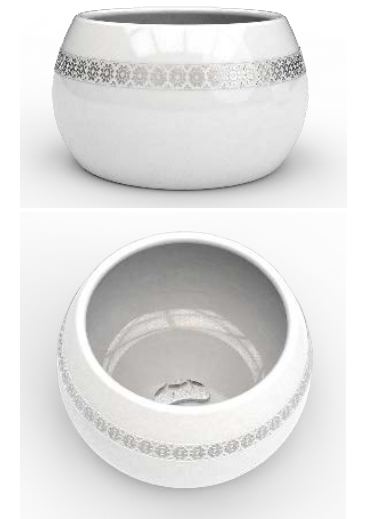

Sample 1
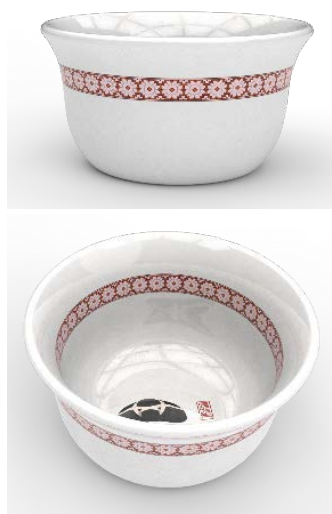

Sample 2
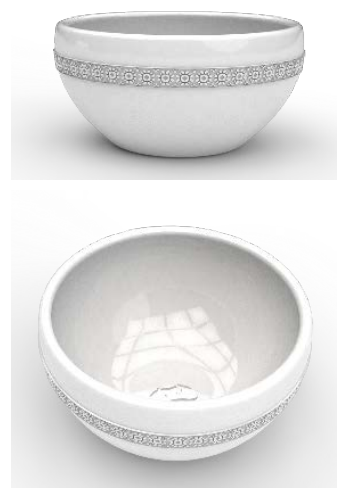

Sample 3

Figure 6. Comparative experiment samples

Then, the sample is psychometrically measured by means of semantic difference method, and the parameters are input into the model to predict the results of the test as shown in Table 3 . The results show that the relative error is between $3 \%$ and $13 \%$, which basically meets the design requirements. 
Table 3. Experimental result

\begin{tabular}{|c|c|c|c|c|}
\hline Items & Error value & Sample 1 & Sample 2 & Sample 3 \\
\hline \multirow{3}{*}{ Beautiful $W_{1}$} & Kansei image score & 1.78 & 1.16 & 1.79 \\
\cline { 2 - 5 } & Model predict score & 1.91 & 1.25 & 1.80 \\
\cline { 2 - 5 } & Relative error & $7.3 \%$ & $7.8 \%$ & $0.6 \%$ \\
\hline \multirow{3}{*}{ Thinness $W_{2}$} & Kansei image score & 2.47 & 1.84 & 1.90 \\
\cline { 2 - 5 } & Model predict score & 2.65 & 1.76 & 2.04 \\
\cline { 2 - 5 } & Relative error & $7.3 \%$ & $-4.3 \%$ & $7.4 \%$ \\
\hline \multirow{3}{*}{ Luxurious $W_{3}$} & Kansei image score & 4.06 & 2.48 & 3.68 \\
\cline { 2 - 5 } & Model predict score & 4.18 & 2.16 & 3.52 \\
\cline { 2 - 5 } & Relative error & $3.0 \%$ & $-12.9 \%$ & $-4.3 \%$ \\
\hline
\end{tabular}

\section{Conclusion}

Aiming at the Kansei image evaluation of cultural products, this study constructs a GA-ELM tool for Kansei image evaluation. The Kansei image scores of three-dimensional samples obtained by network prediction and semantic difference method are compared, and the validity of the model is verified. The study shortens the time of Kansei evaluation of cultural products, improves the design efficiency and achieves the research goal. The results showed that: $a$. The example shows that GA-ELM model is feasible and reliable, and can improve the evaluation efficiency in the process of product creation. $b$. The application level of culture is taken into account in product evaluation, which enriches the design elements of products. The application of neural network in cultural products is preliminarily explored. In the follow-up study, researchers can try to apply the neural network to the study of complex cultural products with multi-design variables and multidimensional Kansei intention assessment.

\section{Acknowledgments}

This work was partially supported by the Guizhou Social Science and Technology Department Project [Qian Ke He SY20113078]

\section{References}

[1] Defu Bao. Product Design Method Studies Based on Cultural Image[D]. Zhejiang University,2017.

[2] Jiang Shan, Gu feng. Prediction of Feature Matching between Customers and Vehicles[J]. Industrial Engineering and Management. 2012, 17(1):108-111.

[3] Zhu Yan, Chen Gang. Head Shape Design of Service Robots on Kansei Engineering and BP Neural Network. PACKAGING ENGINEERING , 2016(14):63-67.

[4] Guo F, Qu Q X, Chen P, et al. Application of Evolutionary Neural Networks on Optimization Design of Mobile Phone Based on User's Emotional Needs[J]. Human Factors in Ergonomics \& Manufacturing, 2016, 26(3):301315.

[5] Mei Yi, Sun Quanlong, Yu Lihua, et al. Aluminum die casting grain size of the prediction method based algorithm GA-ELM[J]. ACTA METALLURGICA SINICA. 2017, 53(9):1125-1132. 
[6] Guo D, Zhang Y, Xiao Z, et al. Common nature of learning between BP-type and Hopfield-type neural networks [J]. Neuro computing, 2015, 167(C):578-586.

[7] Huang G B, Zhu Q Y, Siew C K. Extreme learning machine: Theory and applications [J]. Neuro computing, 2006, 70: 489

[8] Han Yibo. Gas Emission Prediction Based on GA-ELM[J]. Safety in Coal Mines, 2015, 46(4):166-169.

[9] Wang Yinnian. The research and application of genetic algorithm. Jiangnan University.2009.

[10] Qin Qiong, Liu Shujie, Lai Xu, et al. Interpolation of wind speed data in wind farm based on GA optimized ELM neural network[J]. Acta Energiae Solaris Sinica, 2018,39(08):2125-2132.

[11] Hosseini-Hashemi S, Bedroud M, Nazemnezhad R. An exact analytical solution for free vibration of functionally graded circular/annular Mindlin nanoplates via nonlocal elasticity[J]. Composite Structures, 2013, 103(9):108118.

[12] Dong Yenan. Evaluation of Liangzhu Cultural Artifacts Based on Kansei Image[D]. Zhejiang University of Technology,2013.

[13] Pan Weijie, Fan Lingxiu, Lv Jian. Research on product design scheme evaluation for multi-dimensional imagery[J]. Chinese Journal of Engineering Design, 2017, 24(3):250-255. 